\title{
On the Purification of Factor States
}

\author{
S. L. Woronowicz \\ Department of Mathematical Methods of Physics, University of Warsaw, Warsaw, Poland
}

Received February 4, 1972

\begin{abstract}
Let $\mathfrak{A}$ be a $C^{*}$-algebra and $\mathfrak{A}^{\circ}$ be an opposite algebra. Notions of exact and $j$-positive states of $\mathfrak{A}^{\circ} \otimes \mathfrak{A}$ are introduced. It is shown, that any factor state $\omega$ of $\mathfrak{A}$ can be extended to a pure exact $j$-positive state $\tilde{\omega}$ of $\mathfrak{H}^{\circ} \otimes \mathfrak{U}$. The correspondence $\omega \rightarrow \tilde{\omega}$ generalizes the notion of the purifications map introduced by Powers and Størmer. The factor states $\omega_{1}$ and $\omega_{2}$ are quasi-equivalent if and only if their purifications $\tilde{\omega}_{1}$ and $\tilde{\omega}_{2}$ are equivalent.
\end{abstract}

\section{Introduction}

In the recent paper [4] Powers and Størmer investigated the representations of the CAR algebra $\mathfrak{A}(K)$ induced by generalized free states. Let us recall, that $\mathfrak{A}(K)$ is a $C^{*}$-algebra generated by elements $a(f)$ depending linearly on $f$ running over a Hilbert space $K$ and satisfying the canonical anticommutation relations.

Powers and Størmer found that any generalized free state $\omega$ of $\mathfrak{U}(K)$ can be extended to a pure state $\tilde{\omega}$ of $\mathfrak{U}(K \oplus K)$. $\tilde{\omega}$ is given by an explicit formula. They proved that generalized free states $\omega_{1}$ and $\omega_{2}$ give rise to quasiequivalent factorrepresentation if and only if the representations of $\mathfrak{A}(K \oplus K)$ induced by $\tilde{\omega}_{1}$ and $\tilde{\omega}_{2}$ are equivalent. The proof of the last assertion given by Powers and Størmer uses finite dimensional approximations and depends essentially on the particular structure of $\mathfrak{A}(K)$.

In our paper the problem is considered in a more general setting. We prove, that any factor state $\omega$ of a $C^{*}$-algebra $\mathfrak{A}$ admits extension to a pure state $\tilde{\omega}$ of $\mathfrak{Q}^{\circ} \otimes \mathfrak{A}$ (where $\mathfrak{H}^{\circ}$ denotes the opposite $C^{*}$-algebra) and that correspondence $\omega \mapsto \tilde{\omega}$ obeys all the properties mentioned above.

Now $\tilde{\omega}$ is given by no explicit formula, but is characterized by two requirements: $\tilde{\omega}$ should be $j$-positive and exact (for details see Section 1).

The exactness of a state $\tilde{\omega}$ is expressed in terms of the representation induced by $\tilde{\omega}$. The condition is not easy to check, but there are some results, like Araki's duality theorem [1], which make it possible in the case of generalized free states.

Let us note, that the existence of pure non exact states is not clear. It is related to the existence of factorisation $F_{1}, F_{2} \subset B(H)$ such that $F_{1} \neq F_{2}^{\prime}$ (an example of such a factorisation is given in [3]). 
It seems that the state $\tilde{\omega}$ is determined uniquely by $\omega$. The problem is under investigation and results will be presented in a separate paper.

Carrier Hilbert spaces of representations considered in the paper are assumed to be separable, so our results are valid only for such states, which induce (by G.N.S.-construction) representations in separable Hilbert spaces.

\section{Basic Definitions and Results}

Let $\mathfrak{A}$ be a $C^{*}$-algebra with the unity 1 . Replacing the original multiplication rule in $\mathfrak{A}$ by a new one defined by $a \circ b=b a(a, b \in \mathfrak{A})$ we obtain a new $C^{*}$-algebra denoted by $\mathfrak{I}^{\circ}$. The $C^{*}$-algebra $\mathfrak{I}^{\circ}$ is said to be opposite to $\mathfrak{A}$.

For any $a \in \mathfrak{A}, a^{*}$ considered as an element of $\mathfrak{Q}^{\circ}$ will be denoted by $\bar{a}$. It is seen, that

$$
\mathfrak{U} \ni a \mapsto \bar{a} \in \mathfrak{U}^{\circ}
$$

is an antilinear multiplicative and $*$-invariant (i.e. $\left.\bar{a}^{*}=\overline{a^{*}}\right) 1-1$ mapping.

In what follows, we shall consider $\tilde{\mathfrak{U}}=\mathfrak{A}^{\circ} \otimes \mathfrak{A}$ where the tensor product $\otimes$ includes a suitable completion such that $\tilde{\mathfrak{A}}$ becomes a $C^{*}$-algebra. One can check that the formula

$$
j(\bar{a} \otimes b)=\bar{b} \otimes a
$$

defines an antilinear, multiplicative, $*$-invariant involutive (i.e. $j^{2}=$ id) mapping

$$
j: \tilde{\mathfrak{U}} \rightarrow \tilde{\mathfrak{U}}
$$

We adopt the following notation: for any state $\omega$ of a $C^{*}$-algebra $\mathfrak{A}$, $\pi_{\omega}$ will denote a representation of $\mathfrak{A}$ induced by $\omega$ (GNS-construction). The carrier Hilbert space of a representation $\pi$ will be denoted by $H(\pi)$.

Let $\tilde{\omega}$ be a state of $\tilde{\mathfrak{A}}$. The v. Neumann algebras generated by $\left\{\pi_{\tilde{\omega}}(\overline{1} \otimes a): a \in \mathfrak{A}\right\}$ and $\left\{\pi_{\tilde{\omega}}(\bar{a} \otimes 1): a \in \mathfrak{H}\right\}$ will be denoted by $\mathfrak{A}_{\tilde{\omega}}$ and $\mathfrak{U}_{\tilde{\omega}}^{\circ}$ respectively. We have $\left[\mathfrak{U}_{\tilde{\omega}}, \mathfrak{I}_{\tilde{\omega}}^{\circ}\right]=\{0\}$ for the elements $\overline{1} \otimes a$ and $\bar{a} \otimes 1$ commute in $\tilde{\mathfrak{A}}$. We say that $\tilde{\omega}$ is an exact state if and only if $\mathfrak{A}_{\tilde{\omega}}^{\circ}=\mathfrak{A}_{\tilde{\omega}}^{\prime}$ where $\mathscr{A}^{\prime}$ denotes the commutant of a v. Neumann algebra $\mathscr{A}$.

We say, that a state $\tilde{\omega}$ of $\tilde{\mathfrak{U}}$ is $j$-positive if and only if

$$
\tilde{\omega}(\bar{a} \otimes a) \geqq 0
$$

for any $a \in \mathfrak{A}$. It can be easily proved that any $j$-positive state is $j$-invariant (i.e. $\tilde{\omega}(j(\tilde{a}))=\overline{\tilde{\omega}(\tilde{a})}$ for any $\tilde{a} \in \tilde{\mathfrak{Q}})$.

The main results of the paper are contained in the following two theorems: 
Theorem 1.1. Let $\omega$ be a factor state of $\mathfrak{A}$. Then there exists an exact j-positive pure state $\tilde{\omega}$ of $\tilde{\mathfrak{A}}$ such that

$$
\omega(a)=\tilde{\omega}(\overline{1} \otimes a)
$$

for any $a \in \mathfrak{A}$.

Following in Powers' and Størmer's footsteps we say that the state $\tilde{\omega}$ in the Theorem 1.1 is a purification of $\omega$. The usefulness of this notion is clarified by the following

Theorem 1.2. Let $\omega_{1}$ and $\omega_{2}$ be factor states of $\mathfrak{A}$. Then the states $\omega_{1}$ and $\omega_{2}$ are quasiequivalent if and only if their purifications $\tilde{\omega}_{1}$ and $\tilde{\omega}_{2}$ are equivalent.

Let us recall, that states $\omega_{1}$ and $\omega_{2}$ are called equivalent (quasiequivalent) iff representations $\pi_{\omega_{1}}$ and $\pi_{\omega_{2}}$ are equivalent (quasiequivalent).

\section{Exchange Involutions}

Let $\mathscr{A} C B(H)$ be a von Neumann algebra acting on a separable Hilbert space $H$. Antiunitary operator $J$ will be called an exchange involution for $\left(\mathscr{A}, \mathscr{A}^{\prime}\right)$ if and only if $J^{2}=I$ and

$$
J \mathscr{A} J=\mathscr{A}^{\prime}
$$

where $\mathscr{A}^{\prime}$ denotes the commutant of $\mathscr{A}$.

Let $J$ be an exchange involution for $\left(\mathscr{A}, \mathscr{A}^{\prime}\right)$. A vector $x \in H$ is called $(\mathscr{A}, J)$-positive iff

and

$$
J x=x
$$

$$
(x \mid A J A x) \geqq 0
$$

for any $A \in \mathscr{A}$. Assuming that $\mathscr{A}$ is a factor, one can prove that (2.2) is implied by (2.3).

Definition 2.1. An exchange involution $J$ is called positive iff there exists at least one $(\mathscr{A}, J)$-positive vector $x$ such that its central support is equal $I$.

In the last two section we shall prove the following theorems:

Theorem 2.1. Let $\mathscr{A}$ be a $v$. Neumann algebra Any two positive exchange involutions $J_{1}$ and $J_{2}$ for $\left(\mathscr{A}, \mathscr{A}^{\prime}\right)$ are related by the formula:

$$
J_{1}=V J_{2} V^{-1}
$$

where $V$ is an unitary operator belonging to $\mathscr{A}^{\prime}$. 
Theorem 2.2. Let $\omega$ be a normal state of a standard $v$. Neumann algebra $\mathscr{A} \subset B(H)$. Then there exist a positive exchange involution $J$ and $a$ $(\mathscr{A}, J)$-positive vector $x \in H$ such that

$$
\omega(A)=(x \mid A x)
$$

for any $A \in \mathscr{A}$.

Throughout the paper a v. Neumann algebra $\mathscr{A} \subset B(H)$ is said to be standard if and only if it admits a cyclic and separating vector. This terminology differs from that used by Dixmier.

Latter we shall prove (see Lemma 4.1) that any v. Neumann algebra admiting a positive involution is standard.

We shall need a few facts about the so called standard representation of $C^{*}$-algebras.

Let $\pi$ be a factor representation of a $C^{*}$-algebra $\mathfrak{A}$. Then there exists a representation $\tilde{\pi}$ of $\mathfrak{A}$ quasiequivalent to $\pi$ such that the $\mathrm{v}$. Neumann algebra $\tilde{\pi}(\mathfrak{U})^{\prime \prime}$ is standard. It is known, that $\tilde{\pi}$ is the maximal cyclic representation quasiequivalent to $\pi$, i.e. any cyclic representation quasiequivalent to $\pi$ is contained in $\tilde{\pi}$. It follows immediately that $\tilde{\pi}$ is determined uniquely (up to an unitary equivalence). Moreover representations $\pi_{1}$ and $\pi_{2}$ are quasiequivalent iff $\tilde{\pi}_{1}$ and $\tilde{\pi}_{2}$ are equivalent.

Proof of the Theorem 1.1. Let $\omega$ be a factor state of $\mathfrak{U}$. Since $\tilde{\pi}_{\omega}$ is the maximal cyclic representation quasiequivalent to $\pi_{\omega}$, we can choose a vector $x^{\prime} \in H\left(\tilde{\pi}_{\omega}\right)$ such that

$$
\omega(a)=\left(x^{\prime} \mid \tilde{\pi}_{\omega}(a) x^{\prime}\right) .
$$

Let $\mathscr{A}=\tilde{\pi}_{\omega}(\mathfrak{U})^{\prime \prime}$. According to Theorem 2.2 there exist an exchange involution $J$ for $\left(\mathscr{A}, \mathscr{A}^{\prime}\right)$ and a $(\mathscr{A}, J)$-positive vector $x \in H\left(\tilde{\pi}_{\omega}\right)$ such that

$$
\left(x^{\prime} \mid A x^{\prime}\right)=(x \mid A x)
$$

for any $A \in \mathscr{A}$. In particular setting $A=\tilde{\pi}_{\omega}(a)$ we get

$$
\omega(a)=\left(x \mid \tilde{\pi}_{\omega}(a) x\right)
$$

for any $a \in \mathfrak{U}$.

Let us consider the mapping

$$
\tilde{\mathfrak{I}} \ni \bar{b} \otimes a \mapsto J \tilde{\pi}_{\omega}(b) J \tilde{\pi}_{\omega}(a) .
$$

It is seen, that $(2.5)$ defines a representation of $\tilde{\mathfrak{A}}$. This representation is irreducible. Indeed any operator $S$ commuting with $J \tilde{\pi}_{\omega}(b) J \tilde{\pi}_{\omega}(a)$ (for all $a, b \in \mathfrak{A}$ ) commutes with $J \mathscr{A} J$ and $\mathscr{A}$. Remembering that $J \mathscr{A} J=\mathscr{A}^{\prime}$ we get that $S$ belongs to the center of $\mathscr{A}$ and is of the form $\lambda I$ since $\mathscr{A}$ is a factor. Therefore the functional $\tilde{\omega}$ introduced by

$$
\tilde{\omega}(\bar{b} \otimes a)=\left(x \mid J \tilde{\pi}_{\omega}(b) J \tilde{\pi}_{\omega}(a) x\right)
$$


is a pure state of $\tilde{\mathfrak{A}} . \tilde{\omega}$ is a $j$-positive state for the vector $x$ is $(\mathscr{A}, J)$-positive. Taking into account formulae (2.5) and (2.1) we can conclude, that $\tilde{\omega}$ is an exact state. Moreover setting $b=1$ in (2.6) and comparing with (2.4) we get (1.2). This remark ends the proof.

Let $\pi_{\tilde{\omega}}$ be an irreducible representation of $\tilde{\mathfrak{A}}$ defined by an exact $j$-positive pure state $\tilde{\omega}$. It means that there exists a vector $x \in H\left(\pi_{\tilde{\omega}}\right)$ such that

$$
\tilde{\omega}(\bar{b} \otimes a)=\left(x \mid \pi_{\tilde{\omega}}(\bar{b} \otimes a) x\right)
$$

for any $a, b \in \mathfrak{A}$. As in Section 1 we introduce v. Neumann algebras

$$
\begin{aligned}
\mathfrak{P}_{\tilde{\omega}} & =\left\{\pi_{\tilde{\omega}}(\overline{1} \otimes a): a \in \mathfrak{A}\right\}^{\prime \prime} \\
\mathfrak{I}_{\tilde{\omega}}^{\circ} & =\left\{\pi_{\tilde{\omega}}(\bar{a} \otimes 1): a \in \mathfrak{A}\right\}^{\prime \prime} .
\end{aligned}
$$

We mentioned, that any $j$-positive state is $j$-invariant. It follows immediately that there exists an antiunitary operator $J$ acting in $H\left(\pi_{\tilde{\omega}}\right)$ such that $J^{2}=I, J x=x$ and

$$
J \pi_{\tilde{\omega}}(\bar{b} \otimes a) J=\pi_{\tilde{\omega}}(\bar{a} \otimes b) .
$$

Let us note, that $J$ is an exchange involution for $\left(\mathfrak{U}_{\tilde{\omega}}, \mathfrak{H}_{\tilde{\omega}}^{\prime}\right)$. Indeed, taking into account (2.8) and remembering that $\tilde{\omega}$ is an exact state, we have: $J \mathfrak{A}_{\tilde{\omega}} J=\mathfrak{A}_{\tilde{\omega}}^{\circ}=\mathfrak{Q}_{\tilde{\omega}}^{\prime}$.

Moreover the vector $x$ is $\left(\mathfrak{A}_{\tilde{\omega}}, J\right)$-positive because for any $a \in \mathfrak{A}$

$$
\left(x \mid \pi_{\tilde{\omega}}(\overline{1} \otimes a) J \pi_{\tilde{\omega}}(\overline{1} \otimes a) x\right)=\tilde{\omega}(\bar{a} \otimes a) \geqq 0 .
$$

It means that the involution $J$ is positive (note that $\mathfrak{A}_{\tilde{\omega}}$ is a factor and the central support of any non-vanishing vector is equal $I$ ).

Let us consider the following representation

$$
\mathfrak{U} \ni a \mapsto \pi_{\tilde{\omega}}(\overline{1} \otimes a) \in B\left(H\left(\pi_{\tilde{\omega}}\right)\right) .
$$

Taking into account the irreducibility of $\pi_{\tilde{\omega}}$ one can show that (2.9) is a factor representation.

Setting in (2.7) 1 instead of $b$ we get for all $a \in \mathfrak{A}$ :

$$
\omega(a)=\left(x \mid \pi_{\tilde{\omega}}(\overline{1} \otimes a) x\right)
$$

where $\omega$ denotes a state of $\mathfrak{A}$ introduced by (1.2). It means that $\pi_{\omega}$ is equivalent to a subrepresentation of (2.9). It is known that factor representation is quasiequivalent to any subrepresentation. Therefore (2.9) is equivalent to $\tilde{\pi}_{\omega}$ (note that $\pi_{\tilde{\omega}}(\overline{1} \otimes \mathfrak{A})^{\prime \prime} \equiv \mathfrak{A}_{\tilde{\omega}}$ is standard for it admits a positive involution). All representations are considered up to unitary equivalence. Thus we can identify Hilbert spaces $H\left(\pi_{\tilde{\omega}}\right)$ and $H\left(\tilde{\pi}_{\omega}\right)$ in such a way that $\pi_{\tilde{\omega}}(\overline{1} \otimes a)$ become equal to $\tilde{\pi}_{\omega}(a)$ for all $a \in \mathfrak{A}$. 
After this identification $\mathfrak{U}_{\tilde{\omega}}=\tilde{\pi}_{\omega}(\mathfrak{Q})^{\prime \prime}$. Moreover making use of $(2.8)$ we have

$$
\begin{aligned}
\pi_{\tilde{\omega}}(\bar{b} \otimes a) & =J \pi_{\tilde{\omega}}(\overline{1} \otimes b) J \pi_{\tilde{\omega}}(\overline{1} \otimes a) \\
& =J \tilde{\pi}_{\omega}(b) J \tilde{\pi}_{\omega}(a)
\end{aligned}
$$

We compile the results obtained so far

Lemma 2.1. Let $\tilde{\omega}$ be a purification of a factor state $\omega$. Then the representation $\pi_{\tilde{\omega}}$ is equivalent to a representation given by

$$
\tilde{\mathfrak{U}} \ni \bar{b} \otimes a \mapsto J \tilde{\pi}_{\omega}(b) J \tilde{\pi}_{\omega}(a)
$$

where $J$ is a positive exchange involution for $\left(\tilde{\pi}_{\omega}(\mathfrak{H})^{\prime \prime}, \tilde{\pi}_{\omega}(\mathfrak{Q})^{\prime}\right)$.

Proof of the Theorem 1.2. Let $\omega$ and $\omega_{1}$ be quasiequivalent factor states of $\mathfrak{A}$. Then representations $\tilde{\pi}_{\omega}$ and $\tilde{\pi}_{\omega_{1}}$ are equivalent and we may assume that $\tilde{\pi}_{\omega}=\tilde{\pi}_{\omega_{1}}$.

Let $J$ and $J_{1}$ be positive exchange involutions for $\left(\tilde{\pi}_{\omega}(\mathfrak{H})^{\prime \prime}, \tilde{\pi}_{\omega}(\mathfrak{2})^{\prime}\right)$. Then the following representations:

$$
\begin{aligned}
& \tilde{\mathfrak{U}} \ni \bar{b} \otimes a \mapsto J \tilde{\pi}_{\omega}(b) J \tilde{\pi}_{\omega}(a) \\
& \tilde{\mathfrak{U}} \ni \bar{b} \otimes a \mapsto J_{1} \tilde{\pi}_{\omega}(b) J_{1} \tilde{\pi}_{\omega}(a)
\end{aligned}
$$

are equivalent. Indeed, by using Theorems 2.1 we have $J_{1}=V J V^{-1}$ (where $V$ is an unitary element of $\left.\tilde{\pi}_{\omega}(\mathfrak{A})^{\prime}\right)$ and one can check, that

$$
J_{1} \tilde{\pi}_{\omega}(b) J_{1} \tilde{\pi}_{\omega}(a)=V J \tilde{\pi}_{\omega}(b) J \tilde{\pi}_{\omega}(a) V^{-1}
$$

for all $a, b \in \mathfrak{A}$. Now, the assertion of the Theorems 1.2 follows immediately from Lemma 2.1 .

\section{A Generalized Polar Decomposition}

Results derived in this section together with the Tomita's theory [5] provide the main tools for the proofs of the theorems formulated before. In our opinion the results are very interesting in themselves independently of applications presented in the next section.

We shall use the following notation:

$D(A)$ and $R(A)$ will denote the domain and the range of an operator $A$, respectively. If $A$ and $B$ are operators acting in a Hilbert space $H$, then $A B$ will denote their product defined on the domain $D(A B)$ $=\{x \in D(B): B x \in D(A)\}$.

The closure of a preclosed operator $A$ will be denoted by $\bar{A}$. We say that a selfadjoint operator is invertible if and only if 0 is not its eigenvalue.

Let $\Delta$ be an invertible selfadjoint positive operator acting in a Hilbert space $H$ and let $A \in B(H)$. We assume, that domain of $\Delta^{1 / 2} A^{*}$ is dense in $H$. 
It can be easily checked, that $\left(A \Delta^{1 / 2}\right)^{*}=\Delta^{1 / 2} A^{*}$. Therefore, $A \Delta^{1 / 2}$ is a preclosed operator and $\overline{A \Delta^{1 / 2}}=\left(\Delta^{1 / 2} A^{*}\right)^{*}$. According to the well known theorem of von Neumann $\Gamma=\Delta^{1 / 2} A^{*} \overline{A \Delta^{1 / 2}}$ is a positive selfadjoint operator.

Let $x \in D\left(\Delta^{1 / 2}\right)$. Then $x \in D\left(\overline{A \Delta^{1 / 2}}\right)=D\left(\Gamma^{1 / 2}\right)$ and

$$
\left\|\Gamma^{1 / 2} x\right\|=\left\|\overline{A \Delta^{1 / 2}} x\right\|=\left\|A \Delta^{1 / 2} x\right\| \leqq\|A\|\left\|\Delta^{1 / 2} x\right\| .
$$

Therefore there exists an operator $A_{1} \in B(H)$ such that

$$
\Gamma^{1 / 2} x=A_{1} \Delta^{1 / 2} x
$$

for any $x \in D\left(\Delta^{1 / 2}\right)$. Note that $\left\|A_{1}\right\| \leqq\|A\|$. We know, that $D\left(\Delta^{1 / 2}\right)$ is a core of $\overline{A \Delta^{1 / 2}}$. Therefore it is a core of $\Gamma^{1 / 2}$, and Eq. (3.2) shows that

$$
\Gamma^{1 / 2}=\overline{A_{1} \Delta^{1 / 2}} \text {. }
$$

Selfadjointness of $\Gamma^{1 / 2}$ implies immediately that

$$
\overline{A_{1} \Delta^{1 / 2}}=\Gamma^{1 / 2}=\Delta^{1 / 2} A_{1}^{*} .
$$

Among other things it means that $A_{1}^{*} D\left(\Delta^{1 / 2}\right) \subset D\left(\Delta^{1 / 2}\right)$.

Let $x \in D\left(\Delta^{1 / 2}\right)$. The Schwarz inequality for the positive sesquilinear form $\left(\cdot \mid \Delta^{1 / 2} \cdot\right)$ and (3.4) lead to the following relation:

$$
\begin{aligned}
\left|\left(x \mid A_{1}^{n} \Delta^{1 / 2} x\right)\right| & =\left|\left(A_{1}^{* n} x \mid \Delta^{1 / 2} x\right)\right| \leqq\left(x \mid \Delta^{1 / 2} x\right)^{1 / 2}\left(A_{1}^{* n} x \mid \Delta^{1 / 2} A_{1}^{* n} x\right)^{1 / 2} \\
& =\left(x \mid \Delta^{1 / 2} x\right)^{1 / 2}\left(x \mid A_{1}^{2 n} \Delta^{1 / 2} x\right)^{1 / 2} .
\end{aligned}
$$

By repeated use of this sort of inequality we see, that

$$
\left|\left(x \mid A_{1} \Delta^{1 / 2} x\right)\right| \leqq\left(x \mid \Delta^{1 / 2} x\right)^{1 / 2+1 / 4+\cdots+1 / 2^{n}}\left(x \mid A_{1}^{2^{n}} \Delta^{1 / 2} x\right)^{1 / 2^{n}} .
$$

Taking $n \rightarrow \infty$ and remembering that $\left\|A_{1}\right\| \leqq\|A\|$ we get

It means that

$$
\left(x \mid \Gamma^{1 / 2} x\right) \leqq\|A\|\left(x \mid \Delta^{1 / 2} x\right)
$$

$$
\left\|\Gamma^{1 / 4} x\right\| \leqq\|A\|^{1 / 2}\left\|\Delta^{1 / 4} x\right\| .
$$

By repeating the argument leading to the formula (3.3) one can prove, that there exists a bounded operator $A_{2} \in B(H)$ such that $\left\|A_{2}\right\| \leqq\|A\|$ and

$$
\Gamma^{1 / 4}=\overline{A_{2} \Delta^{1 / 4}} .
$$

Now we can formulate our result:

Theorem 3.1. In the situation described above, operators $A_{1}$ and $A_{2}$ belong to the smallest von Neumann algebra generated by $\left\{\Delta^{i s} A \Delta^{-i s}: s \in \mathbb{R}^{1}\right\}$. 
Remark. Note that according to our assumption 0 is not an eigenvalue of $\Delta$ and $\left\{\Delta^{i s}\right\}_{s \in \mathbb{R}^{1}}$ is a well defined one-parameter group of unitary operators.

At first we prove

Lemma. Let $f(\cdot)$ be an analytic function on the right half-plane $\{z: \operatorname{Re} z>0\}$. Assume that

$$
|f(z)| \leqq K e^{M \operatorname{Re} z}
$$

and that $f(n)=0$ for $n=1,2,3, \ldots$. Then $f$ vanishes identically.

Proof. Let us consider the following function:

$$
g(k)=\int_{-\infty}^{+\infty} \frac{f\left(\frac{1}{2}+i x\right)}{\sin \pi\left(\frac{1}{2}+i x\right)} e^{i k x} d x .
$$

It is seen, that $g(\cdot)$ is an analytic function in the strip $|\operatorname{Im} k|<\pi$. On the other hand estimation (3.6) combined with the analytic properties of the integrated function shows, that $g(k)=0$ for $\operatorname{Re} k<-M$. Therefore $g(k) \equiv 0$ and the lemma follows.

Proof of the Theorem. Let $\mathscr{A}$ be the smallest von Neumann algebra generated by $\left\{\Delta^{i s} A \Delta^{-i s}: s \in \mathbb{R}^{1}\right\}$. We have to prove, that $A_{1}, A_{2} \in \mathscr{A}$.

For any operator $B \in \mathscr{A}^{\prime}$ and any integrable function $f$ on $\mathbb{R}^{1}$ we put

$$
B(f)=\int_{-\infty}^{+\infty} \Delta^{i s} B \Delta^{-i s} f(s) d s
$$

where the integral is taken in the strong operator topology. It is seen, that $B(f) \in \mathscr{A}^{\prime}$. We shall assume, that the function $f$ is an entire function such that

$$
\int_{-\infty}^{+\infty}|f(s-i r)| d s \leqq K e^{M|r|}
$$

where $K$ and $M$ are numbers independent of $r \in \mathbb{R}^{1}$.

An example of such a function is given by

$$
f(z)=\int_{-\infty}^{+\infty} e^{i k z} \tilde{f}(k) d k
$$

where $\tilde{f} \in C_{0}^{2}\left(\mathbb{R}^{1}\right)$. In what follows, $f_{r}$ will denote the function $f_{r}(s)=f(s-i r)$.

One can check, that

$$
B(f) \Delta^{r} \subset \Delta^{r} B\left(f_{r}\right)
$$


for any $r \in \mathbb{C}^{1}$. Remembering that $[A, B(f)]=0$ we have

and

$$
B(f) \Delta^{1 / 2} A^{*} \subset \Delta^{1 / 2} B\left(f_{1 / 2}\right) A^{*}=\Delta^{1 / 2} A^{*} B\left(f_{1 / 2}\right)
$$

$$
\begin{aligned}
B(f) \overline{A \Delta^{1 / 2}} & \subset \overline{B(f) A \Delta^{1 / 2}}=\overline{A B(f) \Delta^{1 / 2}} \\
\subset \overline{A \Delta^{1 / 2} B\left(f_{1 / 2}\right)} & =\overline{A \Delta^{1 / 2}} B\left(f_{1 / 2}\right) .
\end{aligned}
$$

Combining these relations we get

and

$$
B(f) \Gamma \subset \Gamma B\left(f_{1}\right)
$$

$$
B(f) \Gamma^{r} \subset \Gamma^{r} B\left(f_{r}\right)
$$

for $r=0,1,2, \ldots$. We are going to prove that the last relation is satisfied for any $r \in \mathbb{C}^{1}$ such that $\operatorname{Re} r \geqq 0$.

To this end, for any $N \in R$ we put $E_{N}=\chi_{\left[0, e^{N}\right]}(\Gamma)$ (where $\chi_{[a, b]}$ is the characteristic function of an interval $[a, b])$ and consider the following bounded operator

$$
F(r)=E_{N} B(f) \Gamma^{r} E_{N}-\Gamma^{r} E_{N} B\left(f_{r}\right) E_{N} .
$$

It is seen, that $F(\cdot)$ is an analytic operator valued function on the right half-plane and that

$$
\|F(r)\| \leqq 2\|B\| K e^{(M+N) \operatorname{Rer}}
$$

for $\operatorname{Re} r>0$. Moreover the relation (3.9) says, that $F(r)=0$ for $r=1,2,3, \ldots$. By using the lemma, one immediately get

$$
E_{N} B(f) \Gamma^{r} E_{N}=\Gamma^{r} E_{N} B\left(f_{r}\right) E_{N}
$$

for any $r$ in the right half-plane. Let $N \rightarrow \infty$. Then $s-\lim E_{N}=I$, $\lim \Gamma^{r} E_{N} x=\Gamma^{r} x$ for any $x \in D\left(\Gamma^{r}\right)$ and the above formula turns into (3.9). Setting in (3.9) $r=1 / 2$ and using formula (3.4) we obtain

$$
B(f) A_{1} \Delta^{1 / 2} \subset \overline{A_{1} \Delta^{1 / 2}} B\left(f_{1 / 2}\right)
$$

By using formula (3.8) we get

$$
\begin{aligned}
B(f) A_{1} \uparrow R\left(\Delta^{1 / 2}\right) & \subset \overline{A_{1} \Delta^{1 / 2}} B\left(f_{1 / 2}\right) \Delta^{-1 / 2} \\
& \subset \overline{A_{1} \Delta^{1 / 2}} \Delta^{-1 / 2} B(f) \subset A_{1} B(f) .
\end{aligned}
$$

Remembering that $A_{1}$ is a bounded operator we have

$$
B(f) A_{1}=A_{1} B(f) .
$$

Let $f(k)$ in (3.7) tends to 1 in a proper way. Then $s-\lim B(f)=B$ and $B A_{1}=A_{1} B$. We have proved that $A_{1}$ commutes with any element of $\mathscr{A}^{\prime}$ and therefore $A_{1} \in \mathscr{A}$. In the same way, setting in (3.9) $r=1 / 4$ one can prove that $A_{2} \in \mathscr{A}$. This ends our proof. 
Let us note that

$$
\left\|A_{1} x\right\|=\|A x\|
$$

for any $x \in H$. Indeed Eqs. (3.1) and (3.2) show, that the above equation is satisfied for any $x \in R\left(\Delta^{1 / 2}\right)$.

Assume now that the ranges $R\left(\Delta^{1 / 2} A^{*}\right)$ and $R(A)$ are dense in $H$. Formula (3.3) shows that $R\left(A_{1}\right)$ is dense in $R\left(\Gamma^{1 / 2}\right)=R\left(\Delta^{1 / 2} A^{*}\right)$. Therefore there exists an unitary operator $W$ such that

$$
A=W A_{1} \text {. }
$$

Theorem 3.1 says, that $W \in \mathscr{A}$.

We view (3.10) as a generalized polar decomposition.

\section{The Proof of the Theorem 2.1}

Lemma 4.1. Let $J$ be a positive exchange involution for $\left(\mathscr{A}, \mathscr{A}^{\prime}\right)$. Then there exists a $(\mathscr{A}, J)$-positive separating and cyclic vector.

Proof. Assume that $x$ is a $(\mathscr{A}, J)$-positive vector. Then

$$
\|A x\|^{2} \leqq\|x\|\|A J A x\|
$$

for any $A \in \mathscr{A}$. Indeed the above inequality can be derived by setting $A_{1}=A^{*} A$ and $A_{2}=I$ in the Schwarz inequality:

$$
\left|\left(x \mid A_{1} J A_{2} x\right)\right| \leqq\left(x \mid A_{1} J A_{1} x\right)^{1 / 2}\left(x \mid A_{2} J A_{2} x\right)^{1 / 2} .
$$

Now let $x$ be the vector appearing in Definition (2.1). By using Zorn's lemma one can find a maximal subset $\left\{x_{0}=x, x_{1}, x_{2}, \ldots\right\} \subset H$ of nonvanishing vectors such that

$1^{\circ} \mathscr{A}^{\prime} x_{k} \perp \mathscr{A}^{\prime} x_{m}$ for $k \neq m$.

$2^{\circ}$ Each $x_{k}$ is of the form $A_{k} J A_{k} x$, where $A_{k}$ is an element of $\mathscr{A}$.

We shall prove that

$$
H=\oplus \overline{\mathscr{A}^{\prime} x_{k}} .
$$

To this end denote by $P$ the orthogonal projection onto $\oplus \overline{\mathscr{A}^{\prime} x_{k}}$. It is seen that $P \in \mathscr{A}$. For any $A \in \mathscr{A}$ and any $k$ we have:

$$
(I-P) A x_{k}=0 \text {. }
$$

Indeed, otherwise $\left\{x_{0}, x_{1}, \ldots\right\}$ could be enlarged by adding the vector $(I-P) A J(I-P) A x_{k}=(I-P) A A_{k} J(I-P) A A_{k} x$, which would be nonvanishing in virtue of (4.1).

Relation (4.3) means that $A x_{k} \in \oplus \overline{\mathscr{A}^{\prime} x_{k}}$. It follows immediately, that $\oplus \overline{\mathscr{A}^{\prime} x_{k}}$ is invariant under all operators from $\mathscr{A}$. Therefore $P \in \mathscr{A}^{\prime}$. 
Moreover $P x=x$. Remembering that the central support of $x$ is equal $I$ we get $P=I$ and (4.2) follows.

Applying the involution $J$ to the both sides of (4.2) we get

$$
H=\oplus \overline{\mathscr{A} x_{k}} \text {. }
$$

Let $y=\Sigma a_{n} x_{n}$, where $a_{k}$ are positive numbers such that the series converges. The formula

$$
(y \mid A J A y)=\sum \sum a_{n} a_{m}\left(x \mid A_{n}^{*} A A_{m} J A_{n}^{*} A A_{m} x\right) \geqq 0,
$$

where $A$ is any element of $\mathscr{A}$, shows that $y$ is $(\mathscr{A}, J)$-positive.

We shall prove that $y$ is a separating and cyclic vector. Indeed denoting by $P_{k}$ the orthogonal projection onto $\overline{\mathscr{A}^{\prime} x_{k}}$ we have $P_{k} \in \mathscr{A}$, $P_{k} y=a_{k} x_{k}$ and (4.4) shows that $\overline{\mathscr{A} y}=H$. Similarly denoting by $P_{k}^{\prime}$ the orthogonal projection onto $\overline{\mathscr{A} x_{k}}$ we have $P_{k}^{\prime} \in \mathscr{A}^{\prime}, P_{k} y=a_{k} x_{k}$ and (4.2) shows that $\overline{\mathscr{A}^{\prime} y}=H$. This ends the proof of the lemma.

Lemma 4.2. Assume that a $v$. Neumann algebra $\mathscr{A} \subset B(H)$ admits a separating and cyclic vector $y \in H$. Then there exists one and only one exchange involution $J$ for $\left(\mathscr{A}, \mathscr{A}^{\prime}\right)$ such that $y$ is $(\mathscr{A}, J)$-positive.

Proof. Let us consider antilinear operators

$$
\begin{array}{ll}
H \ni A y \mapsto A^{*} y \in H & A \in \mathscr{A}, \\
H \ni A^{\prime} y \mapsto A^{\prime *} y \in H & A^{\prime} \in \mathscr{A}^{\prime} .
\end{array}
$$

It is known (see [5]), that these operators are preclosed and that there closures, denoted by $S$ and $F$ respectively, are related by $S^{*}=F$.

Let $J$ be an exchange involution for $\left(\mathscr{A}, \mathscr{A}^{\prime}\right)$ such that $y$ is $(\mathscr{A}, J)$ positive. Then $J y=y$ and $J \mathscr{A} y=\mathscr{A}^{\prime} y$. Moreover for any $A \in \mathscr{A}$ we have:

$$
J F J A y=J F J A J y=J(J A J)^{*} y=A^{*} y=S A y .
$$

It follows immediately from the above relations, that $J F J=S$ and $F J=J S$.

Let

$$
\Delta^{1 / 2}=J S .
$$

It is seen, that $\Delta^{1 / 2}$ is a closed linear operator and that $\mathscr{A} x$ is its core. The above equation can be rewritten as follows:

$$
S=J \Delta^{1 / 2} .
$$

We shall prove that (4.8) is the polar decomposition of $S$, i.e. that $\Delta^{1 / 2}$ is a positive selfadjoint operator. Indeed, for any $A \in \mathscr{A}$ the matrix element

$$
\left(A y \mid \Delta^{1 / 2} A y\right)=(A y \mid J S A y)=\left(y \mid A^{*} J A^{*} y\right)
$$


is nonnegative, since $y$ is $(\mathscr{A}, J)$-positive. Moreover

$$
\left(\Delta^{1 / 2}\right)^{*}=(J S)^{*}=S^{*} J=F J=J S=\Delta^{1 / 2} .
$$

Now, taking into account the uniqueness at the polar decomposition (4.8) we can conclude, that there exists of most one exchange involution $J$ for $\left(\mathscr{A}, \mathscr{A}^{\prime}\right)$ such that $y$ is $(\mathscr{A}, J)$-positive.

On the other hand, according to the Tomita's theory [5] the antiunitary operator $J$ introduced by the polar decomposition (4.8) is an exchange involution for $\left(\mathscr{A}, \mathscr{A}^{\prime}\right)$. Moreover Eq. (4.9) shows that the vector $y$ is $(\mathscr{A}, J)$-positive for the operator $\Delta^{1 / 2}$ in (4.8) should be positive. This ends the proof of the lemma.

Let $y$ be separating and cyclic vector. The exchange involution $J$ such that $y$ is $(\mathscr{A}, J)$-positive will be denoted by $J_{y}$. Assume that $W$ is an unitary operator belonging to $\mathscr{A}$. Then

$$
J_{W y}=W J_{y} W^{-1} .
$$

Indeed $W J_{y} W^{-1}$ is an exchange involution for $\left(\mathscr{A}, \mathscr{A}^{\prime}\right)$ and $W y$ is evidently $\left(\mathscr{A}, W J_{y} W^{-1}\right)$-positive.

Let $y \in H$ and $A \in \mathscr{A}$. Assume that $y$ and $A y$ are both cyclic and separating vectors. Then $R(A)$ contains $\mathscr{A}^{\prime} A y$ and is dense in $H$.

Lemma 4.3. In the situation described above, the exchange involutions $J_{y}$ and $J_{A y}$ are related by

$$
J_{A y}=W J_{y} W^{-1}
$$

where $W$ is an unitary operator belonging to $\mathscr{A}$.

Proof. Let $\Delta$ be the positive selfadjoint operator introduced by (4.7), where $J$ should be replaced by $J_{y}$. For any $B \in \mathscr{A}$ we have $B y \in D\left(\Delta^{1 / 2} A^{*}\right)$ and

$$
\Delta^{1 / 2} A^{*} B y=J_{y} S A^{*} B y=J_{y} B^{*} A y .
$$

It shows that $D\left(\Delta^{1 / 2} A^{*}\right) \supset \mathscr{A} y$ and $R\left(\Delta^{1 / 2} A^{*}\right) \supset J_{y} \mathscr{A} A y$. Therefore the domain and the range of $\Delta^{1 / 2} A^{*}$ are dense in $H$.

According to the previous sections we can introduce bounded operators $A_{1}, A_{2}$ and an unitary operator $W$ such that:

$$
\begin{gathered}
\left(\Delta^{1 / 2} A^{*} \overline{A \Delta^{1 / 2}}\right)^{1 / 2}=\overline{A_{1} \Delta^{1 / 2}} \\
\left(\Delta^{1 / 2} A^{*} \overline{A \Delta^{1 / 2}}\right)^{1 / 4}=\overline{A_{2} \Delta^{1 / 4}}=\Delta^{1 / 4} A_{2}^{*} \\
A=W A_{1} .
\end{gathered}
$$

One of the main results of Tomita theory says that $\Delta^{i s} \mathscr{A} \Delta^{-i s}=\mathscr{A}$ for any real $s$. Therefore in virtue of Theorem 3.1, we obtain $A_{1}, A_{2}, W \in \mathscr{A}$. 
Taking into account definitions (4.7) and (4.5) we have:

$$
\begin{aligned}
A_{2} J_{y} A_{2} y & =A_{2} J_{y} S A_{2}^{*} y=A_{2} \Delta^{1 / 2} A_{2}^{*} y \\
& =\left(A_{2} \Delta^{1 / 4}\right)\left(\Delta^{1 / 4} A_{2}^{*}\right) y=\left(\Delta^{1 / 2} A^{*} \overline{A \Delta^{1 / 2}}\right)^{1 / 2} y=A_{1} y
\end{aligned}
$$

since $\Delta^{1 / 2} y=J_{y} S y=y$. It follows that the vector $A_{1} y$ is $\left(\mathscr{A}, J_{y}\right)$-positive. Indeed, by using (4.13) one can check, that $J_{y} A_{1} y=A_{1} y$ and

$$
\left(A_{1} y \mid B J_{y} B A_{1} y\right)=\left(y \mid A_{2}^{*} B A_{2} J_{y} A_{2}^{*} B A_{2} y\right) \geqq 0
$$

for any $B \in \mathscr{A}$. According to Lemma $4.2 J_{A_{1} y}=J_{y}$ and (4.11) is implied by (4.12) and (4.10). So, the lemma is proven.

Now, we can prove the Theorem 2.1 .

Let $J_{1}$ and $J_{2}$ be positive exchange involutions for $\left(\mathscr{A}, \mathscr{A}^{\prime}\right)$. In virtue of Lemmas 4.1 and 4.2 there exist vectors $y_{1}$ and $y_{2}$, both separating and cyclic such that $J_{1}=J_{y_{1}}$ and $J_{2}=J_{y_{2}}$.

It can be proved, that there exists a separating and cyclic vector $y \in H$ such that

$$
\left(y \mid A^{\prime} y\right)=\left(y_{1} \mid A^{\prime} y_{1}\right)+\left(y_{2} \mid A^{\prime} y_{2}\right)
$$

for any $A^{\prime} \in \mathscr{A}^{\prime}$. To this end one can use the well known properties of faithful normal states of standard von Neumann algebras. These properties are mentioned in the next section.

Setting $A^{\prime *} A^{\prime}$ instead of $A^{\prime}$ in (4.14) we get $\left\|A^{\prime} y_{1}\right\| \leqq\left\|A^{\prime} y\right\|$. Remembering that $\mathscr{A}^{\prime} y$ is dense in $H$ one can introduce an operator $A_{1} \in B(H)$ such that

$$
A_{1} A^{\prime} y=A^{\prime} y_{1}
$$

for any $A^{\prime} \in \mathscr{A}^{\prime}$. There is no problem to check that $A_{1} \in \mathscr{A}$. Moreover,

$$
y_{1}=A_{1} y \text {. }
$$

In the same way one can prove, that

$$
y_{2}=A_{2} y,
$$

where $A_{2}$ is an operator from $\mathscr{A}$.

Now, by using Lemma 4.3 we get

$$
J_{1}=W J_{2} W^{-1},
$$

where $W$ is an unitary operator belonging to $\mathscr{A}$. Evidently the above formula can be rewritten as follows:

where

$$
J_{1}=V J_{2} V^{-1},
$$

$$
V=J_{2} W^{-1} J_{2} \in \mathscr{A}^{\prime} .
$$

The last formula ends the proof. 


\section{The Proof of the Theorem 2.2}

We start with the following

Lemma 5.1. Let $J$ be an exchange involution for $\left(\mathscr{A}, \mathscr{A}^{\prime}\right)$ and $y$ be a $(\mathscr{A}, J)$-positive separating and cyclic vector. Assume that $x=P y=P^{\prime} y$, where $P$ and $P^{\prime}$ are projection operators belonging to $\mathscr{A}$ and $\mathscr{A}^{\prime}$ respectively. Then the vector $x$ is $(\mathscr{A}, J)$-positive.

Proof. We keep the notation introduced in Section 4. It is seen, that

$$
\begin{gathered}
S x=S P y=P^{*} y=x \\
F x=F P^{\prime} y=P^{\prime *} y=x .
\end{gathered}
$$

It shows that $\Delta x=F S x=x$ and $\Delta^{-1 / 2} x=x$. Therefore $J x=S \Delta^{-1 / 2} x=x$. Remembering that $y$ is a separating vector and using the relation

$$
J P^{\prime} J y=J P^{\prime} y=J x=x=P y
$$

we get $P=J P^{\prime} J$. It follows that $P^{\prime} J P^{\prime}=P J P$ and for any $A \in \mathscr{A}$ :

$$
\begin{aligned}
(x \mid A J A x) & =\left(P^{\prime} P y \mid A J A P^{\prime} P y\right)=\left(y \mid P A P^{\prime} J P^{\prime} A P y\right) \\
& =(y \mid P A P J P A P y) \geqq 0
\end{aligned}
$$

since $y$ is $(\mathscr{A}, J)$-positive. It means that $x$ is $(\mathscr{A}, J)$-positive.

Let us recall, that a normal state $\varrho$ of a v. Neumann algebra $\mathscr{A}$ is said to be faithful if and only if

$$
\left(\varrho\left(A^{*} A\right)=0\right) \Rightarrow(A=0)
$$

for any $A \in \mathscr{A}$. It is known, that any v. Neumann algebra (acting in a separable Hilbert space) admits a faithful state and that any faithful normal state of a standard v. Neumann algebra $\mathscr{A} \subset B(H)$ can be represented by a separating and cyclic vector $y \in H$.

Let $\omega$ be a normal state of a v. Neumann algebra $\mathscr{A}$. The support of $\omega$ (see [2], I. $\S 4.6$ ) will be denoted by P.P is a projection operator belonging to $\mathscr{A}$ such that

for any $A \in \mathscr{A}$.

$$
\begin{gathered}
\left(\omega\left(A^{*} A\right)=0\right) \Rightarrow\left(P A^{*} A P=0\right) \\
\omega(P A P)=\omega(A)
\end{gathered}
$$

Now we are in position to prove the Theorem (2.2). Let $\omega$ be a normal state of a standard v. Neumann algebra $\mathscr{A}$. We introduce

$$
\varrho(A)=\omega(A)+\sigma((I-P) A(I-P))
$$

where $A \in \mathscr{A}, P$ is the support of $\omega$ and $\sigma$ is a faithful state of $\mathscr{A}$. One can check, that $\varrho$ is a faithful state. Therefore for any $A \in \mathscr{A}$ :

$$
\omega(A)+\sigma((I-P) A(I-P))=(y \mid A y)
$$


where $y \in H$ is a separating and cyclic vector. In virtue of Lemma 4.2 there exists an exchange involution $J$ for $\left(\mathscr{A}, \mathscr{A}^{\prime}\right)$ such that $y$ is $(\mathscr{A}, J)$ positive.

Setting $(I-P) A P$ instead of $A$ in (5.2) and using (5.1) we get

$$
(y \mid(I-P) A P y)=0
$$

for any $A \in \mathscr{A}$. It means that

$$
(I-P) y \perp \overline{\mathscr{A} P y} \text {. }
$$

Let $P^{\prime}$ denote the orthogonal projection onto $\overline{\mathscr{A} P y}$. Evidently $P^{\prime} \in \mathscr{A}^{\prime}$ and (5.3) shows that $P^{\prime} y=P y$. According to Lemma $5.1 x=P y$ is a $(\mathscr{A}, J)$-positive vector. Moreover, setting $P A P$ instead of $A$ in (5.2) and using (5.1) we get

$$
\omega(A)=(y \mid P A P y)=(x \mid A x)
$$

This ends the proof of the Theorem 2.2.

\title{
References
}

1. Araki, H.: A latties of von Neumann algebras associated with the quantum theory of a free Bose field. J. Math. Phys. 4, 1345-1362 (1963).

2. Dixmier,J.: Les algebras d'operateurs dans l'espace Hilbertien. Paris: GauthierVillars 1969.

3. Murray,F.J., von Neumann,J.: On rings of operators I. Ann. Math. 37, 116-229 (1936).

4. Powers, R.T., Størmer,E.: Free states of the canonical anticommutation relations. Commun. math. Phys. 16, 1-33 (1970).

5. Takesaki, M.: Tomita's theory of modular Hilbert algebras and its applications. Lecture Notes in Mathematics 128. Berlin-Heidelberg-New York: Springer 1970.

\author{
S. L. Woronowicz \\ Department of Mathematical \\ Methods of Physics \\ University of Warsaw \\ Hoza 74 \\ Warsaw, Poland
}


